\title{
An Investigation of Solving Third-Order Nonlinear Ordinary Differential Equation in Complex Domain by Generalising Prelle-Singer Method
}

\author{
Ali K. Joohy (ID, Ghassan A. Al-Juaifri, and Mohammed S. Mechee \\ Faculty of Computer Science and Mathematics, University of Kufa, Kufa, Iraq \\ Correspondence should be addressed to Ali K. Joohy; alijoohy@gmail.com
}

Received 1 June 2019; Accepted 20 February 2020; Published 17 March 2020

Academic Editor: Mostafa Eslami

Copyright (c) 2020 Ali K. Joohy et al. This is an open access article distributed under the Creative Commons Attribution License, which permits unrestricted use, distribution, and reproduction in any medium, provided the original work is properly cited.

\begin{abstract}
A method to solve a family of third-order nonlinear ordinary complex differential equations (NLOCDEs) - nonlinear ODEs in the complex plane-by generalizing Prelle-Singer has been developed. The approach that the authors generalized is a procedure of obtaining a solution to a kind of second-order nonlinear ODEs in the real line. Some theoretical work has been illustrated and applied to several examples. Also, an extended technique of generating second and third motion integrals in the complex domain has been introduced, which is conceptually an analog to the motion in the real line. Moreover, the procedures of the method mentioned above have been verified.
\end{abstract}

\section{Introduction}

In the last five decades, fascinating methods have been made in identifying nonlinear integrable dynamical systems in the real line. In particular, different methods have been developed or modified to investigate innovative integrable cases and experience the potential dynamics that could be related to the finite-dimensional nonlinear dynamical systems, which is defined on the real numbers [1]. The most extensively mathematical tools that are being used to solve ODEs are Painleve Analysis [1, 2], Direct Method, [3] Lie Symmetry Analysis $[1,4]$, Noether's theorem [1, 4], Direct Linearization [5], $\lambda$-symmetries, adjoint symmetries, and Jacobi last multiplier technique $[6,7]$.

Thirty years ago, in the community of DEs, systems of DEs and integrable dynamical systems, Prelle and Singer [8] had made a particularly powerful approach for solving a kind of first-order nonlinear ODEs in the real line, and it obtains a solution which consists of elementary functions on the real line. In the present work, the authors are moving a step forward to generalize one particular method, namely, Prelle-Singer method, and present a related procedure on the complex plane; the authors have generalized a class of nonlinear ODEs [9], in which it has a real interval of definition. Then, they construed a class of (NLOCDE (Nonlinear Ordinary Complex Differential Equations (NLOCDE, where the general solution to the mentioned))) is an algebraic combination of complex elementary functions that are analytic on a particular region in the complex plane.

The central concept of the Prelle-Singer scheme is that if the real addressed system of first-order nonlinear ODEs has a solution that was built from real elementary functions, then this method assures that this solution is going to exist. Earlier in 2001, Duarte et al. published a paper [10], in which they extended the method that had been introduced by Prelle and Singer in 1983 [8] to be relevant for solving the secondorder nonlinear ODEs. The instant strategy is based on the assumption, that is, if a complex elementary solution exists for the given second-order nonlinear OCDEs then there exists at least one elementary first integral in the complex domain $I(z, w, w \prime)$, in which all its derivatives are complex rational functions of $z, w$, and $w /$. Duarte et al. had deduced first integrals for some classes of ODEs for the first time.

Recently, in the literature, there has been a generalization to the theory given in [10], and a solution to a class of nonlinear oscillator equations [11]. Also, there were very 
early contributions to the subject where solutions of ODEs are constructed as a fraction of functions and the method that is used, roughly speaking, slightly similar to the methods in the literature, as applied mathematics enrichment [12]. In the last work [13], the authors generalized the concept of ODEs and investigated some applications in physics and engineering based on the ideas in [14]. In this paper, the authors enlarged the theory of Duarte that he had presented in [10] and made it applicable on third-order nonlinear OCDEs and procure a relation which attaches complex integrals of motion (integrals in the complex plan) analog with the complex integrating factors. They also illustrated the theory with an example. Furthermore, they showed that one could generate the demanded number of complex integrals of motion (motion integral in the complex plane) analog from the first complex integral of motion. The design of the paper is as follows: in Section 2, authors developed the theory of the extended Prelle-Singer method to make it applicable to the third-order ordinary nonlinear CDEs. In Sections 1-4, the theoretical definitions have been shown; in Section 5, authors considered an example and constructed complex integrals of motion. In Section 6, a procedure in which one can generate second and third complex integrals of motion from the first integral for a class of equations is described. In Section 7, the theory was verified with the implementations of some examples that were considered. In Section 8, a Hamiltonian system of dispersive water waves has been solved. Finally, the conclusion was located in Section 9.

\section{Preliminaries}

This section shows fundamental definitions about the differential equations in the complex domain [15-19].

Definition 1. A complex differential equation is an equation which contains derivatives of a complex analytic function of one or more independent variables:

$$
w=F\left(z_{1}, z_{2}, \ldots, z_{n}\right),
$$

where $z_{1}, z_{2}, \ldots, z_{n}$ are complex dependent variables. The general form of complex differential equations is

$$
f\left(z, w, \frac{\partial w}{\partial z_{i}}, \frac{\partial^{2} w}{\partial z_{i} \partial z_{j}}, \frac{\partial^{n} w}{\partial z_{i} \partial z_{j} \ldots \partial z_{n}}\right)=0,
$$

and it will be called $\mathrm{CDE}$ as stands for it, and $w=F\left(z_{1}, z_{2}, \ldots, z_{n}\right)$ is the solution for the CDE.

Definition 2. The order of the CDE (CDE stands for complex differential equation) is the highest derivative in that CDE.

Definition 3. The degree of the $\mathrm{CDE}$ is the power of the highest derivative in that $\mathrm{CDE}$.

Remark 1. The complex differential equation has two types: ordinary complex differential equations and partial complex differential equations.
(1) Ordinary complex differential equations (OCDE's) have one dependence and one independence complex variables

(2) Partial complex differential equations (PCDE's) have more than one independence complex variable

Remark 2. In this work, the authors concentrate the study on the ordinary complex differential equations.

\section{Ordinary Complex Differential Equations}

The general form of ordinary complex differential equations is $[20-22]$

$$
\frac{d^{n} w}{d z^{n}}=f\left(z, w, w^{\prime}, w^{(2)}, \ldots, w^{(n-1)}\right) .
$$

Definition 4. The particular solution is a general solution with a specified value for the constant $C$.

Definition 5. Consider the OCDEs that have the general form [23]

$$
\frac{d^{n} w}{d z^{n}}=f\left(z, w, w^{\prime}, w^{(2)}, \ldots, w^{(n-1)}\right) .
$$

Equation (4) is called the linear ordinary complex differential equation when (4) is linear in $w$ and its drivatives.

Definition 6. The ordinary complex differential equation is called nonlinear complex differential equation when it is nonlinear [24].

\section{Analysis of Prelle-Singer Method for Third- Order OCDEs}

Consider the kind of third-order nonlinear OCDEs of the standard form [25]

$$
\frac{d^{3} w}{d z^{3}}=\frac{G\left(z, w, w^{\prime}, w^{\prime \prime}\right)}{H\left(z, w, w^{\prime}, w^{\prime \prime}\right)} H \neq 0,
$$

where $G$ and $H$ are polynomials with the constant coefficients in the complex field.

Let's presume that OCDE (5) concedes the first integral $I\left(z, w, w^{\prime}, w^{\prime \prime}\right)=C$ when $C$ is a complex constant in the family solutions. Hence, the total differentiation is

$$
d I=\frac{\partial I}{\partial z} d z+\frac{\partial I}{\partial w} d w+\frac{\partial I}{\partial w^{\prime}} d w^{\prime}+\frac{\partial I}{\partial w^{\prime \prime}} d w^{\prime \prime}=0 .
$$

By rephrasing (5) in the scheme

$$
\frac{G}{H} d z-d w^{\prime \prime}=0
$$

and appending null expressions $\left(S w^{\prime} \mathrm{d} z-S \mathrm{~d} w=S w^{\prime}-\right.$ $S(\mathrm{~d} w / \mathrm{d} z)=w^{\prime}-w^{\prime}=0 F w^{\prime \prime} \mathrm{d} z-F \mathrm{~d} w=F w^{\prime \prime}-F\left(\mathrm{~d} w^{\prime} / \mathrm{d} z\right)$ $\left.=w^{\prime \prime}-w^{\prime \prime}=0\right)$, 


$$
\begin{aligned}
& S\left(z, w, w^{\prime}, w^{\prime \prime}\right) w^{\prime} d z-S\left(z, w, w^{\prime}, w^{\prime \prime}\right) d w \\
& F\left(z, w, w^{\prime}, w^{\prime \prime}\right) w^{\prime \prime} d z-F\left(z, w, w^{\prime}, w^{\prime \prime}\right) d w^{\prime}
\end{aligned}
$$

to equation (7), we obtain

$$
\left(\frac{G}{H}+S w^{\prime}+F w^{\prime \prime}\right) d z-S d w-F d w^{\prime}-d w^{\prime \prime}=0,
$$

Thence, on the solutions, equations (6) and (9) must be equivalent. Multiplying equation (9) by the complex factor of integration function $R\left(z, w, w^{\prime}, w^{\prime \prime}\right)$, for the CDE (5), we obtain

$$
\mathrm{d} I=R\left(\phi+S w^{\prime}+F w^{\prime \prime}\right) \mathrm{d} z-R S \mathrm{~d} w-R F \mathrm{~d} w^{\prime}-R \mathrm{~d} w^{\prime \prime}=0,
$$

where $\phi \equiv(P / Q)$.

Comparing equations (6) with (10), we get the equations

$$
\begin{aligned}
I_{z} & =R\left(\phi+S w^{\prime}+F w^{\prime \prime}\right), \\
I_{w} & =-R S, \\
I_{w^{\prime}} & =-R F, \\
I_{w^{\prime \prime}} & =-R .
\end{aligned}
$$

The compatibility conditions $I_{z w}=I_{w z}, I_{z w^{\prime}}=I_{w^{\prime} z}$, $I_{z w^{\prime \prime}}=I_{w^{\prime \prime} z}$, and $I_{w w^{\prime}}=I w^{\prime} w, I_{w^{\prime} w^{\prime \prime}=I_{w^{\prime \prime} w^{\prime}}}$, between equation (11) equip us with the following:

$$
\begin{aligned}
D[S] & =-\phi_{w}+S \phi_{w^{\prime \prime}}+F S, \\
D[F] & =-\phi_{w^{\prime}}+F \phi_{w^{\prime \prime}}+F^{2}-S, \\
D[R] & =-R\left(F+\phi_{w^{\prime \prime}}\right), \\
R_{w^{\prime}} S & =-R S_{w^{\prime}}+R_{x} F+R F_{x}, \\
R_{w^{\prime}} & =R_{w^{\prime \prime}} F+R F_{w^{\prime \prime}}, \\
R_{w} & =R_{w^{\prime \prime}} S+R S_{w^{\prime \prime}},
\end{aligned}
$$

where $D$ is the total differential complex operator:

$$
D=\frac{\partial}{\partial z}+w^{\prime} \frac{\partial}{\partial w}+w^{\prime \prime} \frac{\partial}{\partial w^{\prime}}+\phi \frac{\partial}{\partial w^{\prime \prime}}
$$

It should be noted that equations (12)-(17) form an overdetermined system for the undisclosed complex functions, $S, F$, and $R$.

Having solved them through substituting the $\phi=G / H$ into equations (12) and (13), the output will be a system of CDEs of the unknowns complex functions $S$ and $F$. By solving the equalisations, one can capture expressions for the null forms $S$ and $F$. Once $F$ is grasped, then (14) turns to be the determining equation for the function $R$. Through working out, the latter one can occupy an explicit construction for $R$. Momentarily, the complex functions
$R, F$, and $S$ have to satisfy the additional constraints (15)-(17).

Nevertheless, as soon as a cooperative solution satisfies every equation that has been gained suddenly, the complex functions $R, F$, and $S$ fix the first integral $I\left(z, w, w^{\prime}, w^{\prime \prime}\right)$ by the relation

$$
I=\zeta_{1}-\zeta_{2}-\zeta_{3}-\int\left(R+\frac{\mathrm{d}}{\mathrm{d} w^{\prime \prime}}\left(\zeta_{1}-\zeta_{2}-\zeta_{3}\right)\right) \mathrm{d} w^{\prime \prime},
$$

where

$$
\begin{aligned}
& \zeta_{1}=\int R\left(\phi+S w^{\prime}+F w^{\prime \prime}\right) \mathrm{d} z, \\
& \zeta_{2}=-\int\left(R S+\frac{\mathrm{d}}{\mathrm{d} w^{\prime}} \zeta_{1}\right) \mathrm{d} w, \\
& \zeta_{3}=-\int\left(R F+\frac{\mathrm{d}}{\mathrm{d} w^{\prime \prime}}\left(\zeta_{1}+\zeta_{2}\right)\right) \mathrm{d} w^{\prime \prime} .
\end{aligned}
$$

Equation (19) can be procured straightforwardly via performing integrating to equation (11). Promptly, substitute the expressions of $\phi, R, F$, and $S$ into equation (19) and attain the integrals when the related integrals of motion can be gained.

\section{Implementation}

In this section, we illustrate the theory that had been developed and has been shown in Section 4 [26].

\subsection{Example 1}

5.1.1. A: Determination of Integration Factors and Null Terms. Consider the following equation:

$$
w^{\prime \prime \prime}=\frac{6 z\left(w^{\prime \prime}\right)^{3}}{w^{\prime 2}}+\frac{6 w^{\prime \prime 2}}{w^{\prime}} .
$$

Substituting $\phi=\left(\left(6 z\left(w^{\prime \prime}\right)^{3}\right) /\left(w^{\prime 2}\right)\right)+\left(6 w^{\prime \prime 2} / w^{\prime}\right)$ into (12)-(14), we obtain

$$
\begin{aligned}
& \frac{\partial S}{\partial z}+w^{\prime} \frac{\partial S}{\partial w}+w^{\prime \prime} \frac{\partial S}{\partial w^{\prime}}+\left(\frac{6 z\left(w^{\prime \prime}\right)^{3}}{w^{\prime 2}}+\frac{6 w^{\prime \prime 2}}{w^{\prime}}\right) \frac{\partial S}{\partial w^{\prime \prime}} \\
& =S\left(\frac{18 z\left(w^{\prime \prime}\right)^{3}}{w^{\prime 2}}+\frac{12 w^{\prime \prime 2}}{w^{\prime}}+F\right) \\
& \frac{\partial F}{\partial z}+w^{\prime} \frac{\partial F}{\partial w}+w^{\prime \prime} \frac{\partial F}{\partial w^{\prime}}+\left(\frac{6 z\left(w^{\prime \prime}\right)^{3}}{w^{\prime 2}}+\frac{6 w^{\prime \prime 2}}{w^{\prime}}\right) \frac{\partial F}{\partial w^{\prime \prime}} \\
& =\frac{12 z w^{\prime \prime}}{w^{\prime 3}}+\frac{6 w^{\prime \prime 2}}{w^{\prime 2}}+F\left(\frac{18 z w^{\prime \prime 2}}{w^{\prime 2}}+\frac{12 w^{\prime \prime}}{w^{\prime}}\right)+F^{2}-S
\end{aligned}
$$




$$
\begin{aligned}
\frac{\partial R}{\partial z} & +w^{\prime} \frac{\partial R}{\partial w}+w^{\prime \prime} \frac{\partial R}{\partial w^{\prime}}+\left(\frac{6 z w^{\prime \prime 3}}{w^{\prime w^{\prime 2}}}+\frac{6 w^{\prime \prime^{2}}}{w^{\prime}}\right) \frac{\partial R}{\partial w^{\prime \prime}} \\
& =-R\left(F+\frac{18 z w^{\prime \prime 2}}{w^{\prime 2}}+\frac{12 w^{\prime \prime}}{w^{\prime}}\right) .
\end{aligned}
$$

As mentioned in Section 4, first, we have solved system (11) and obtained explicit forms for the functions $S$ and $F$. We observe that, for this particular example, $S=0$ is a simple solution for equation (22). So, we consider the implication of this solution.

Now substituting $S=0$ into equation (23), we get a firstorder differential equation for $F$, namely,

$$
\begin{aligned}
\frac{\partial F}{\partial z} & +w^{\prime} \frac{\partial F}{\partial w}+w^{\prime \prime} \frac{\partial F}{\partial w^{\prime}}+\left(\frac{6 z w^{\prime \prime 3}}{w^{2}}+\frac{6 w^{\prime \prime 2}}{w^{\prime}}\right) \frac{\partial F}{\partial w^{\prime \prime}} \\
& =\frac{12 z w^{\prime \prime}}{w^{\prime 3}}+\frac{6 w^{\prime \prime 2}}{w^{\prime 2}}+F\left(\frac{18 z w^{\prime \prime 2}}{w^{\prime 2}}+\frac{12 w^{\prime \prime}}{w^{\prime}}\right)+F^{2}
\end{aligned}
$$

To find the solution to equation (25), one could approach $F$ of the pattern

$$
F=\frac{a\left(z, w, w^{\prime}\right)+b\left(z, w, w^{\prime}\right) w^{\prime \prime}+c\left(z, w, w^{\prime}\right) w^{\prime \prime 2}}{d\left(z, w, w^{\prime}\right)+e\left(z, w, w^{\prime}\right) w^{\prime \prime}+f\left(z, w, w^{\prime \prime}\right) w^{\prime \prime 2}}
$$

where $a, b, c, d, e$, and $f$ are arbitrary functions of $z, w$, and $w^{\prime}$.

Having substituted equation (26) into (25) and made the coefficients of the same power of $w^{\prime \prime}$ vanish, one achieves a family of PCDEe (PCDE stands for Partial Complex Differential Equations) with respect to the variables $a, b, c, d, e$, and $f$.

By solving, one quickly obtains

$$
\begin{aligned}
& F_{1}=-\frac{6 z w^{\prime \prime 2}+3 w^{\prime} w^{\prime \prime}}{w^{\prime 2}}, \\
& F_{2}=-\frac{6 z w^{\prime \prime 2}+4 w^{\prime} w^{\prime \prime}}{w^{\prime 2}} .
\end{aligned}
$$

Replacing the complex relations $F_{1}$ and $F_{2}$ with (24) and working out the latter, one will be able to gain a precise pattern of the complex factor function $R$.

First, let us consider $F_{1}$, and the corresponding equation for $R$ becomes

$$
\begin{array}{r}
\frac{\partial R}{\partial z}+w^{\prime} \frac{\partial R}{\partial w}+w^{\prime \prime} \frac{\partial R}{\partial w^{\prime}}+\left(\frac{6 z w^{\prime \prime 3}}{w^{\prime 2}}+\frac{6 w^{\prime \prime 2}}{w^{\prime}}\right) \frac{\partial R}{\partial w^{\prime \prime}} \\
=R\left(\frac{6 z w^{\prime \prime 2}+3 w^{\prime} w^{\prime \prime}}{w^{\prime 2}}-\frac{18 z w^{\prime 2}}{w^{\prime 2}}-\frac{12 w^{\prime \prime}}{w^{\prime}}\right),
\end{array}
$$

To solve (28) again, one has to make ansatz. We assume the following form for $R$, that is,

$$
R=\frac{A(z, w, w,)+B\left(z, w, w^{\prime}\right) w^{\prime \prime}+C\left(z, w, w^{\prime}\right) w^{\prime \prime 2}}{D\left(z, w, w^{\prime}\right)+E\left(z, w, w^{\prime}\right) w^{\prime \prime}+F\left(z, w, w^{\prime \prime}\right) w^{\prime \prime}}
$$

where $A, B, C, D, E$, and $F$ are arbitrary functions of $z, w$, and $w \prime$.

Now substituting equation (29) into (28) and equating the coefficients of differential power of $w^{\prime \prime}$ to zero and solving the resultant equations, we obtain the following expressions for $R$, namely,

$$
R_{1}=\frac{w^{\prime 3}}{w^{\prime \prime 2}}
$$

Similarly, replacing the term of $F_{2}$ into (28) and solving the resultant equation with the same type of ansatz (29), we arrive at

$$
R_{2}=\frac{w^{\prime 4}}{w^{\prime \prime 2}}
$$

To compile, we reach the subsequent pattern of solutions (with ansatz (27) and (29) for equations (22)-(24)):

$$
\begin{aligned}
& \left(S_{1}, F_{1}, R_{1}\right)=\left(0,-\frac{6 z w^{\prime \prime 2}+3 w^{\prime} w^{\prime \prime}}{w^{\prime 2}}, \frac{w^{\prime 3}}{w^{\prime \prime 2}}\right), \\
& \left(S_{2}, F_{2}, R_{2}\right)=\left(0,-\frac{6 z w^{\prime 2}+4 w^{\prime} w^{\prime \prime}}{w^{\prime 2}}, \frac{w^{\prime 4}}{w^{\prime \prime 2}}\right) .
\end{aligned}
$$

At the stage above, we have left three more equations unsolved, that is,

$$
\begin{aligned}
R_{w^{\prime}} S & =-R S_{w^{\prime}}+R_{w} F+R F_{w} \\
R_{w^{\prime}} & =R_{w^{\prime \prime}} F+R F_{w^{\prime \prime}} \\
R_{w} & =R_{w^{\prime \prime}} S+R S_{w^{\prime \prime}} .
\end{aligned}
$$

However, one can quickly verify that solution (32) also satisfies extraconstraints (33)-(35). As a result, (32) forms compatible solutions for systems (12)-(17) with $\phi$ given in (21). Finally, we note that, in the above, we considered only a trivial solution $S=0$ for equation (25) and derived the corresponding forms of $F$ and $R$. However, in the choice $S \neq 0$, systems (22) and (23) become a coupled equation in the unknowns $F$ and $S$. To solve this system, as we did previously, let us make an ansatz:

$$
\begin{aligned}
& S=\frac{\bar{a}(z, w, w,)+\bar{b}\left(z, w, w^{\prime}\right) w^{\prime \prime}+\bar{c}\left(z, w, w^{\prime}\right) w^{\prime \prime 2}}{\bar{d}\left(z, w, w^{\prime}\right)+\bar{e}\left(z, w, w^{\prime}\right) w^{\prime \prime}+\bar{f}\left(z, w, w^{\prime \prime}\right) w^{\prime 2^{2}}}, \\
& F=\frac{a(z, w, w,)+b\left(z, w, w^{\prime}\right) w^{\prime \prime}+c\left(z, w, w^{\prime}\right) w^{\prime \prime 2}}{d\left(z, w, w^{\prime}\right)+e\left(z, w, w^{\prime}\right) w^{\prime \prime}+f\left(z, w, w^{\prime \prime}\right) w^{\prime \prime 2}},
\end{aligned}
$$


in which the coefficients are arbitrary functions in $\left(z, w, w^{\prime}\right)$. Substituting equations (36) and (37) in equations (22) and (23) and solving the resultant equations, we arrive at

$$
\begin{aligned}
& F_{3}=-\frac{6 z w^{\prime \prime 2}+2 w^{\prime} w^{\prime \prime}}{w^{\prime 2}} \\
& S_{3}=\frac{2 w^{\prime 2}}{w^{\prime 2}}
\end{aligned}
$$

which in turn forces $R$ to stay of the form

$$
R_{3}=\frac{w^{\prime 2}}{w^{\prime \prime 2}}
$$

Expressions (38) and (39) satisfy extraconstraints (33)-(35), so the functions

$$
\left(S_{3}, F_{3}, R_{3}\right)=\left(\frac{2 w^{\prime \prime 2}}{w^{\prime 2}},-\frac{z w w^{\prime \prime^{2}}+2 w^{\prime} w^{\prime \prime}}{w^{\prime 2}}, \frac{w^{\prime 2}}{w^{\prime 2}}\right)
$$

also form a compatible solution for equations (12)-(17) with $\phi$ given in (21).

5.1.2. B: Integrals of Motion. The determined functions $\left(S_{i}, F_{i}, R_{i}\right), i=1,2,3$, we can go on to determine the related motion integrals, and by substituting the expressions $\left(S_{i}, F_{i}, R_{i}\right), i=1,2,3$, into (19) separately and evaluating the integrals, we obtain

$$
\begin{aligned}
& I_{1}=3 z w^{\prime} w^{2}+\frac{w^{\prime 3}}{w^{\prime \prime}}, \\
& I_{2}=2 z w^{\prime 3}+\frac{w^{\prime 4}}{w^{\prime \prime}}, \\
& I_{3}=2 z-6 z w^{\prime}-\frac{w^{\prime 2}}{w^{\prime \prime}},
\end{aligned}
$$

respectively. It can easily be checked that $I^{\prime} \mathrm{s}, i=1,2,3$, are constants on the complex solutions, that is, $\left(\mathrm{d} I_{i} / \mathrm{d} z\right)=0$, $i=1,2,3$. From the integrals, $I_{1}, I_{2}$, and $I_{3}$, we can deduce the general complex solution for equation (21). For example, solving equation (41) for $w^{\prime \prime}$ and substituting into equations (42) and (43), we obtain

$$
\begin{array}{r}
z w^{\prime 3}-I_{1} w^{\prime}+I_{2}=0, \\
3 z w^{\prime 2}+\left(I_{3}-2 w\right) w^{\prime}+I_{1}=0,
\end{array}
$$

after algebraically combining these equations to eliminate, and we obtain a functional relation between $w$ and $z$ as

$$
\begin{aligned}
& 3 z\left(I_{1}\left(I_{3}-2 w\right)-9 z I_{2}\right)^{2}+I_{1}\left(\left(I_{3}-2 w\right)^{2}-12 z I_{1}\right)^{2} \\
& -\left(I_{3}-2 w\right)\left(I_{1}\left(I_{3}-2 w\right)-9 z I_{2}\right)\left(\left(I_{3}-2 w\right)^{2}-12 z I_{1}\right)=0 .
\end{aligned}
$$

We mention that expression (45) was derived from a different point of view using the generalized factors in [27].

\section{Method of Generating Complex Integrals of Motion Analogue}

In Section 5, the authors have derived the motion integrals $I_{i}$, $i=1,2,3$, by building a sufficient number of integrating factors. Beautifully, one can also generate the needed amount of motion integrals from the first integral itself. For example, for the thirdorder system that is presented as equation (21), one can create $I_{2}$ and $I_{3}$ from $I_{1}$ itself. In the following work, we are going to illustrate our ideas.

Let us assume there is a first integral for the third-order equation (5) of the form

$$
I_{1}=F_{1}\left(z, w, w^{\prime}, w^{\prime \prime}\right)
$$

Let us assume there are two factors to the function $F_{1}$ in which it can be represented in the structure of two different multiplied complex functions such that one includes a perfect differentiable function $(\mathrm{d} / \mathrm{d} z) G_{1}\left(z, w, w^{\prime}\right)$ and the other function $G_{2}\left(z, w, w^{\prime}, w^{\prime \prime}\right)$, that is,

$$
I_{1}=F_{1}\left(\frac{1}{G_{2}\left(z, w, w^{\prime}, w^{\prime \prime}\right)} \frac{\mathrm{d}}{\mathrm{d} z} G_{1}\left(z, w, w^{\prime}\right)\right),
$$

constructing the complex function $G_{1}\left(z, w, w^{\prime}\right)$ as a new dependent variable and the integral of $G_{2}\left(z, w, w^{\prime}, w^{\prime \prime}\right)$ over time as a new independent variable, namely,

$$
\begin{aligned}
& w=G_{1}\left(z, w, w^{\prime}\right), \\
& z=\int_{0}^{z} G_{2}\left(z \prime, w, w^{\prime}, w^{\prime \prime}\right) \mathrm{d} z \prime .
\end{aligned}
$$

One can represent (47) to be exhibited as

$$
\widehat{I}_{1}=\frac{\mathrm{d} z}{\mathrm{~d} m} \frac{\mathrm{d} n}{\mathrm{~d} z}
$$

Integrating (49), we obtain

$$
n=\widehat{I}_{1} m+I_{2}
$$

where $I_{2}$ is an integration constant. In other words,

$$
I_{2}=n-\widehat{I}_{1} m
$$

Rewriting $n$ and $m$ with respect to the old variables $z, w, w^{\prime}$, and $w^{\prime \prime}$ and replacing $I_{1}$ by its explicit form, one can get an exact form for $I_{2}$. Interestingly, one can represent the first integral $I_{1}$ in form (47) in more than one way, say,

$$
I_{1}=F_{1}\left(\frac{1}{\widehat{G}_{2}\left(z, w, w^{\prime}, w^{\prime \prime}\right)} \frac{\mathrm{d}}{\mathrm{d} z} \widehat{G}_{1}\left(z, w, w^{\prime}\right)\right),
$$

\section{Applications}

To illustrate the abovementioned ideas, we consider the same example (vide equation (21)) discussed in Section 5. In particular, we consider one of the complex integrals and generate the other two through our procedure.

Let us first consider (41), that is, 


$$
I_{1}=3 z w^{\prime} w^{2}+\frac{w^{\prime 3}}{w^{\prime \prime}}
$$

and generate $I_{2}$ and $I_{3}$ from (53). Rewriting (53) in form (47), we obtain

$$
I_{1}=-\frac{1}{w^{\prime \prime}} \frac{\mathrm{d}}{\mathrm{d} z}\left(-z w \prime^{3}\right)=\frac{\mathrm{d} z}{\mathrm{~d} m} \frac{\mathrm{d} w}{\mathrm{~d} z}=\frac{\mathrm{d} n}{\mathrm{~d} m}
$$

so that

$$
\begin{aligned}
& n=-z w^{\prime 3} \\
& m=-w^{\prime}
\end{aligned}
$$

Integrating (54), we obtain

$$
n=I_{1} m+I_{2} \Rightarrow I_{2}=n-I_{1} m \text {. }
$$

Rewriting $n$ and $m$ in terms of the old variables exerting expression (55) and replacing $I_{1}$ by expression (53), we arrive at

$$
I_{2}=2 z\left(w^{\prime}\right)^{3}+\frac{\left(w^{\prime}\right)^{4}}{w^{\prime \prime}}
$$

which is exactly similar to the equation we have derived (vide equation (42)) earlier through the Prelle-Singer method.

Now, to generate $I_{3}$ from $I_{1}$, we rewrite the functions in form (47) but with different latter $\bar{n}$ and $\bar{m}$, namely,

$$
I_{1}=-\frac{\left(w^{\prime}\right)^{2}}{w^{\prime \prime}} \frac{\mathrm{d}}{\mathrm{d} z}\left(2 w-3 z w^{\prime}\right)=\frac{\mathrm{d} z}{\mathrm{~d} \bar{m}} \frac{\mathrm{d} \bar{n}}{\mathrm{~d} z}=\frac{\mathrm{d} \bar{n}}{\mathrm{~d} \bar{m}}
$$

so that

$$
\bar{n}=2 w-3 z w^{\prime} \bar{m}=w^{\prime},
$$

Integrating (59), we obtain

$$
\bar{n}=I_{1} \bar{m}+I_{3} \Rightarrow I_{3}=\bar{n}-I_{1} \bar{m} .
$$

Substituting (59) and (53) into (60), we obtain

$$
I_{3}=2 w-6 z w^{\prime}-\frac{\left(w^{\prime}\right)^{2}}{w^{\prime \prime}}
$$

which exactly agrees with (43). Similarly, we can derive $I_{1}$ and $I_{2}$ from $I_{3}$ and $I_{1}$ and $I_{3}$ from $I_{2}$. As the scheme is the same as the one given above in the following, we provide only the essential steps.

Consider $I_{2}$ as follows:

$$
I_{2}=2 z\left(w^{\prime}\right)^{3}+\frac{\left(w^{\prime}\right)^{3}}{w^{\prime \prime}}
$$

and generate $I_{1}$ and $I_{3}$ from the former. Rewrite the above in the form

$$
I_{2}=-\frac{\left(w^{\prime}\right)^{2}}{w^{\prime \prime}} \frac{\mathrm{d}}{\mathrm{d} z}\left(z\left(w^{\prime}\right)^{2}\right)
$$

Moreover, repeating the abovementioned steps, one gets the first integral (41). On the contrary, the expression $I_{2}$ in the form

$$
I_{2}=-\frac{\left(w^{\prime}\right)^{3}}{w^{\prime \prime}} \frac{\mathrm{d}}{\mathrm{d} z}\left(w-2 z w^{\prime}\right)
$$

leads us to the third integral (43).

\section{Implementation Arising in Physics}

The physical model of describing the dispersive water waves as a system of third-order OCDE is one of the most important implementations of CDEs, and the method of Prelle-Singer plays a significant role to find the integrating factor and eventually the solution of such systems. The physical model finally has the homogeneous nonlinear system [28]:

$$
B H=0,
$$

for some $H$, and $B$ is a Hamiltonian operator of the complex PDEs and

$$
\begin{aligned}
H & =(K, L)^{T}, \\
\frac{\partial^{3}}{\partial z^{3}}(L) & =\Phi_{1}\left(z, L, K, L_{z}, K_{z}, L_{z z}, K_{z z}\right), \\
\frac{\partial^{3}}{\partial z^{3}}(K) & =\Phi_{2}\left(z, L, K, L_{z}, K_{z}, L_{z z}, K_{z z}\right) .
\end{aligned}
$$

For example, system (66) satisfies a first integral which has the form

$$
I\left(z, L, K, L_{z}, K_{z}, L_{z z}, K_{z z}\right)=C,
$$

where it is constant on the solutions. So, the total differentiation will be

$$
\begin{aligned}
\mathrm{d} I & =I_{z} \mathrm{~d} z+I_{L} \mathrm{~d} L+I_{k} \mathrm{~d} K+I_{L_{z}} \mathrm{~d} L_{z}+I_{K_{z}} \mathrm{~d} K_{z} \\
& +I_{L_{z z}} \mathrm{~d} L_{z z}+I_{K_{z z}} \mathrm{~d} K_{z z}=0 .
\end{aligned}
$$

Then, we can write

$$
\begin{aligned}
\Phi_{1} \mathrm{~d} z-\mathrm{d} L_{z z} & =0, \\
\Phi_{2} \mathrm{~d} z-\mathrm{d} K_{z z} & =0 .
\end{aligned}
$$

By adding the null terms in (69),

$$
\begin{aligned}
& \left(\Phi_{1}+S_{1} L_{z}+S_{2} K_{z}+M_{1} L_{z z}+M_{2} K_{z z}\right) \mathrm{d} z \\
& \quad-S_{1} \mathrm{~d} L-S_{2} \mathrm{~d} K-M_{1} \mathrm{~d} L_{z}-M_{2} \mathrm{~d} K_{z}-\mathrm{d} L_{z z}=0, \\
& \left(\Phi_{2}+U_{1} L_{z}+U_{2} K_{z}+N_{1} L_{z z}+N_{2} K_{z z}\right) \mathrm{d} z \\
& \quad-U_{1} \mathrm{~d} L-U_{2} \mathrm{~d} K-N_{1} \mathrm{~d} L_{z}-N_{2} \mathrm{~d} K_{z}-\mathrm{d} L_{z z}=0 .
\end{aligned}
$$

Now, multiplying (70) by the integrating factor $R_{1}$ and (71) by the integrating factor $R_{2}$, where $R_{1}=R_{1}\left(z, L, K, L_{z}, K_{z}, L_{z z}, K_{z z}\right)$ and $R_{2}=R_{2}\left(z, L, K, L_{z}\right.$, $K_{z}, L_{z z}, K_{z z}$ ), we get the result as 


$$
\begin{aligned}
\mathrm{d} I= & R_{1}\left(\Phi_{1}+S L_{z}+M L_{z z}\right) \mathrm{d} z+R_{2}\left(\Phi_{2}+U K_{z}+N K_{z z}\right) \mathrm{d} z \\
& -R_{1} S \mathrm{~d} L-R_{2} U \mathrm{~d} K-R_{1} M \mathrm{~d} L_{z}-R_{2} N \mathrm{~d} K_{z} \\
& -R_{1} \mathrm{~d} L_{z z}-R_{2} \mathrm{~d} K_{z z}=0
\end{aligned}
$$

where $S=\left(\left(R_{1} S_{1}+R_{2} U_{1}\right) / R_{1}\right), \quad U=\left(\left(R_{1} S_{2}+R_{2} U_{2}\right) / R_{2}\right)$, $M=\left(\left(R_{1} M_{1}+R_{2} N_{2}\right) / R_{1}\right)$, and $N=\left(\left(R_{1} M_{2}+R_{2} N_{2}\right) / R_{2}\right)$.

When we compare the abovementioned equations with equation (72), we obtain

$$
\begin{aligned}
I_{z} & =R_{1}\left(\Phi_{1}+S L_{z}+M L_{z z}\right)+R_{2}\left(\Phi_{2}+U K_{z}+N K_{z z}\right), \\
I_{L} & =-R_{1} S, \\
I_{K} & =-R_{2} U, \\
I_{L_{z}} & =-R_{1} M, \\
I_{K_{z}} & =-R_{2} N, \\
I_{L_{z z}} & =-R_{1}, \\
I_{K_{z z}} & =-R_{2} .
\end{aligned}
$$

By using the conditions, we obtain the determining equations:

$$
\begin{aligned}
& D\left[R_{1}\right]=-\left(R_{1} \Phi_{1 L_{z z}}+R_{2} \Phi_{2 L_{z z}}+R_{1} M\right) \\
& D\left[R_{2}\right]=-\left(R_{1} \Phi_{1 K_{z z}}+R_{2} \Phi_{2 K_{z z}}+R_{2} N\right) \\
& D[S]=S \Phi_{1 L_{z z}}+\left(\frac{S R_{2}}{R_{1}}\right) \Phi_{2 L_{z z}}+M S-\Phi_{1 L}-\left(\frac{R_{2}}{R_{1}}\right) \Phi_{2 L}
\end{aligned}
$$

$$
D[U]=U \Phi_{2 K_{z z}}+\left(\frac{U R_{2}}{R_{1}}\right) \Phi_{2 K_{z z}}+N U-\Phi_{2 K}-\left(\frac{R_{2}}{R_{1}}\right) \Phi_{1 K},
$$

$$
D[M]=M \Phi_{1 L_{z z}}+\left(\frac{M R_{2}}{R_{1}}\right) \Phi_{2 L_{z z}}+M^{2}-\Phi_{1 L_{z}}-\left(\frac{R_{2}}{R_{1}}\right) \Phi_{2 L_{z}}-S
$$

$$
D[N]=N \Phi_{2 K_{z z}}+\left(\frac{N R_{1}}{R_{2}}\right) \Phi_{1 K_{z z}}+N^{2}-\Phi_{2 K_{z}}-\left(\frac{R_{1}}{R_{2}}\right) \Phi_{1 K_{z}}-U
$$

$$
\begin{array}{r}
R_{1 K_{z}} M+R_{1} M_{K_{z}}=R_{2 L_{z}} N+R_{2} N_{L_{z}}, \\
R_{1 L}=R_{1 L_{z z}} S+R_{1} S_{L_{z z}}, \\
R_{1 K_{z}} S+R_{1} S_{K_{z}}=R_{2 L} N+R_{2} N_{L}, \\
R_{1 K}=R_{2 L_{z z}} U+R_{2} U_{L_{z z}}, \\
R_{2 K_{z}} U+R_{2} U_{K_{z}}=R_{2 K} N+R_{2} N_{K},
\end{array}
$$

$$
\begin{aligned}
R_{1 L_{z}} & =R_{1 L_{z z}} M+R_{1} M_{L_{z z}}, \\
R_{1 L_{z}}+S+R_{1} S_{L_{z}} & =R_{1 L} M+R_{1} M_{L}, \\
R_{1 K_{z}}=R_{1 K_{z}} & =R_{2 L_{z z}} N+R_{2} N_{L_{z z}}, \\
R_{2 L_{z}} U+R_{2} U_{L_{z}} & =R_{1} M_{K}+M R_{1 K}, \\
R_{2 L} & =R_{1 K_{z z}} S+R_{1} S_{K_{z z}}, \\
R_{1 K} S+R_{1} S_{K}= & R_{2 L} U+R_{2} U_{L}, \\
R_{2 K}= & R_{2 K_{z z}} U+R_{2} U_{K_{z z}}, \\
R_{2 L_{z}} & =R_{1 K_{z z}} M+R_{1} M_{K_{z z}}, \\
R_{2 K_{z}} & =R_{2 K_{z z}} N+R_{2} N_{K_{z z}}, \\
R_{1 K_{z z}} & =R_{2 L_{z z}} .
\end{aligned}
$$

When the parts of the solutions $R_{1}, R_{2}, S, U, M$, and $N$ are found, then the integral can be built by the substitution of all the expressions in (73) and find the integration for the result, that is,

$$
\begin{aligned}
I & =r_{1}+r_{2}+r_{3}+r_{4}+r_{5}+r_{6} \\
& -\int\left(R_{2}+\frac{\partial}{\partial K_{z z}}\left(r_{1}+r_{2}+r_{3} r_{4}+r_{5}+r_{6}\right)\right) \mathrm{d} K_{z z},
\end{aligned}
$$

where

$$
\begin{aligned}
& r_{1}=\int\left(R_{1}\left(\Phi_{1}+S L_{z}+M L_{z z}\right)+R_{2}\left(\Phi_{2}+U K_{z}+N K_{z z}\right)\right) \mathrm{d} z, \\
& r_{2}=-\int\left(R_{1} S+\frac{\partial}{\partial L}\left(r_{1}\right)\right) \mathrm{d} L, \\
& r_{3}=-\int\left(R_{2} U+\frac{\partial}{\partial K}\left(r_{1}+r_{2}\right)\right) \mathrm{d} K, \\
& r_{4}=-\int\left(R_{1} M+\frac{\partial}{\partial L_{z}}\left(r_{1}+r_{2}+r_{3}\right)\right) \mathrm{d} L_{z}, \\
& r_{5}=-\int\left(R_{2} N+\frac{\partial}{\partial K_{z}}\left(r_{1}+r_{2}+r_{3}+r_{4}\right)\right) \mathrm{d} K_{z}, \\
& r_{6}=-\int\left(R_{1}+\frac{\partial}{\partial L_{z z}}\left(r_{1}+r_{2}+r_{3}+r_{4}+r_{5}\right)\right) .
\end{aligned}
$$

To determine the integrating factors $R_{1}$ and $R_{2}$, we represent the determining equations $(74)-(79)$ as two equations: 


$$
\begin{aligned}
D^{3}\left[R_{1}\right]= & D^{2}\left[R_{1} \Phi_{1 L_{z z}}+R_{2} \Phi_{2 L_{z z}}\right]-D\left[R_{1} \Phi_{1 L_{z}}+R_{2} \Phi_{2 L_{z}}\right] \\
& +R_{1} \Phi_{1 L}+R_{2} \Phi_{2 L}=0, \\
D^{3}\left[R_{2}\right]= & D^{2}\left[R_{1} \Phi_{1 K_{z z}}+R_{2} \Phi_{2 K_{z z}}\right]-D\left[R_{1} \Phi_{1 K_{z}}+R_{2} \Phi_{2 K_{z}}\right] \\
& +R_{1} \Phi_{1 K}+R_{2} \Phi_{2 K}=0,
\end{aligned}
$$

where

$$
D=\frac{\partial}{\partial z}+L_{z} \frac{\partial}{\partial L}+K_{z} \frac{\partial}{\partial K}+L_{z z} \frac{\partial}{\partial L_{z}}+K_{z z} \frac{\partial}{\partial K_{z}}+\Phi_{1} \frac{\partial}{\partial L_{z z}}+\Phi_{2} \frac{\partial}{\partial K_{z z}} .
$$

The determining equations (97) and (98) represents a system of linear of PDEs in $R_{1}$ and $R_{2}$. Substituting the terms $\Phi_{1}$ and $\Phi_{2}$ and their derivatives into (97) and (98) and solving them, we can obtain expressions for the integrating factor $R_{1}$ and $R_{2}$. After finding the $R_{s}$, the functions $(S, U, M$, and $N)$ can be fixed through relations (76)-(79). After one finds $R_{1}$, $R_{2}, S, U, M$, and $N$, then he has to make sure that they satisfy conditions (80)-(94). So, the set $\left(S, U, M, N, R_{1}, R_{3}\right)$ that satisfies equations (74)-(94) will form the wanted solution and the integral which has form (95).

\section{Conclusion}

In this work, the authors investigated a new method for solving third-order OCDEs through the technique of modified Prelle-Singer. The process was not straightforward for [10] but had some new theoretical aspects that have several advantages. The research illustrated the theory with some implementations. It also introduced a new way of generating second and third complex integrals of motion analog from the first complex integral and demonstrated the idea with the same example that considered previously. The authors solved a system of thirdorder NLOCDE that arises in physics, precisely in dispersive water waves. The advantage of this work is to find a rational solution to the ordinary nonlinear differential equation in the complex domain and to apply the theories of solving ODEs to the complex plane. As an initial step, extended Prelle-Singer was widened to be applicable on the models in physics and engineering that imply DEs in complex domain as a significant part. In future work, the road will be paved to generalize and investigate the other methods on the complex domain.

\section{Data Availability}

No data were used to support this study.

\section{Conflicts of Interest}

The authors declare that they have no conflicts of interest.

\section{References}

[1] M. Lakshmanan and S. Rajasekar, Nonlinear Dynamics: Integrability, Chaos and Patterns, Springer, Berlin, Heidelberg, Germany, 2003.
[2] A. Ramani, B. Grammaticos, and T. Bountis, "The Painlevé property and singularity analysis of integrable and non-integrable systems," Physics Reports, vol. 180, no. 3, pp. 159-245, 1989.

[3] J. Hietarinta, "Direct methods for the search of the second invariant," Physics Reports, vol. 147, no. 2, pp. 87-154, 1987.

[4] M. Lakshmanan and R. Sahadevan, "Painlevé analysis, lie symmetries, and integrability of coupled nonlinear oscillators of polynomial type," Physics Reports, vol. 224, no. 1-2, pp. 1-93, 1993.

[5] N. Euler and M. Euler, "Sundman symmetries of nonlinear second-order and third-order ordinary differential equations," Journal of Nonlinear Mathematical Physics, vol. 11, no. 3, pp. 399-421, 2004.

[6] R. Mohanasubha, V. K. Chandrasekar, M. Senthilvelan, and M. Lakshmanan, "Interplay of symmetries, null forms, darboux polynomials, integrating factors and Jacobi multipliers in integrable second-order differential equations," Proceedings of the Royal Society A: Mathematical, Physical and Engineering Sciences, vol. 470, no. 2163, p. 20130656, 2014.

[7] R. Mohanasubha, V. K. Chandrasekar, M. Senthilvelan, and M. Lakshmanan, "Interconnections between various analytic approaches applicable to third-order nonlinear differential equations," Proceedings of the Royal Society A: Mathematical, Physical and Engineering Sciences, vol. 471, no. 2176, p. 20140720, 2015.

[8] M. J. Prelle and M. F. Singer, "Elementary first integrals of differential equations," Transactions of the American Mathematical Society, vol. 279, no. 1, p. 215, 1983.

[9] S.-M. Jung and J. Roh, "The linear differential equations with complex constant coefficients and Schrödinger equations," Applied Mathematics Letters, vol. 66, pp. 23-29, 2017.

[10] L. G. S. Duarte, S. E. S. Duarte, L. A. C. P. d. Mota, and J. E. F. Skea, "Solving second-order ordinary differential equations by extending the prelle-singer method," Journal of Physics A: Mathematical and General, vol. 34, no. 14, pp. 3015-3024, 2001.

[11] V. K. Chandrasekar, M. Senthilvelan, and M. Lakshmanan, "New aspects of integrability of force-free Duffing-van der Pol oscillator and related nonlinear systems," Journal of Physics A: Mathematical and General, vol. 37, no. 16, pp. 4527-4534, 2004.

[12] J. Avellar, M. S. Cardoso, L. G. S. Duarte, and L. A. C. P. da Mota, "Dealing with rational second order ordinary differential equations where both darboux and lie find it difficult: the s-function method," Computer Physics Communications, vol. 234, pp. 302-314, 2019.

[13] A. K. Joohy, Ordinary Complex Differential Equations with Applications in Science and Engineering, 2018, http://arxiv. org/abs/1805.03201.

[14] E. Hille, Ordinary Differential Equations in the Complex Domain, Courier Corporation, Chelmsford, MA, USA, 1997.

[15] B. Guo, S. Peng, X. Hu, and P. Xu, "Complex-valued differential operator-based method for multi-component signal separation," Signal Processing, vol. 132, pp. 66-76, 2017.

[16] A. A. Aswhad and A. F. Jaddoa, "The approximate solution of newell whitehead segel and Fisher equations using the adomian decomposition method," 2016, http://arxiv.org/abs/ 1602.04084 .

[17] H. H. Bauschke and P. L. Combettes, "Hilbert spaces," in Convex Analysis and Monotone Operator Theory in Hilbert Spaces, Springer, Berlin, Heidelberg, Germany, 2017.

[18] J. Baylis, "Advanced calculus of a single variable by Tunc Geveci, pp. 382, £37.99 (hard), ISBN 978-3-31927-806-3, also 
available as e-book, Springer Verlag (2016)," The Mathematical Gazette, vol. 101, no. 551, pp. 367-368, 2017.

[19] M. S. Mechee, G. A. Al-Juaifri, and A. K. Joohy, "Modified homotopy perturbation method for solving generalized linear complex differential equations," Applied Mathematical Sciences, vol. 11, no. 51, pp. 2527-2540, 2017.

[20] G. Fano and S. Blinder, Twenty-First Century Quantum Mechanics: Hilbert Space to Quantum Computers: Mathematical Methods and Conceptual Foundations, Springer, Berlin, Heidelberg, Germany, 2017.

[21] S. Lang, Complex Analysis, vol. 103, Springer Science and Business Media, Berlin, Heidelberg, Germany, 2013.

[22] Z. Huang, W. Zhu, D. P. Arovas, J.-X. Zhu, and A. V. Balatsky, "Invariance of topological indices under Hilbert space truncation," Physical Review Letters, vol. 120, no. 1, Article ID 016403, 2018

[23] Y. Sibuya, Ordinary Differential Equations in Complex Domain, John Wiley \& Sons, New York, NY, USA, 1976.

[24] Y. Sibuya, "Linear differential equations in the complex domain: problems of analytic continuation," Journal of the American Mathematical Society, vol. 82, 2008.

[25] L. G. S. Duarte, S. E. S. Duarte, L. A. C. P. da Mota, and J. E. F. Skea, "An extension of the Prelle-Singer method and a Maple implementation," Computer Physics Communications, vol. 144, no. 1, pp. 46-62, 2002.

[26] V. K. Chandrasekar, S. N. Pandey, M. Senthilvelan, and M. Lakshmanan, "Application of extended Prelle-Singer procedure to the generalized modified Emden type equation," Chaos, Solitons \& Fractals, vol. 26, no. 5, pp. 1399-1406, 2005.

[27] V. K. Chandrasekar, M. Senthilvelan, and M. Lakshmanan, "On the complete integrability and linearization of certain second-order nonlinear ordinary differential equations," Proceedings of the Royal Society A: Mathematical, Physical and Engineering Sciences, vol. 461, no. 2060, pp. 2451-2477, 2005.

[28] P. R. Gordoa, A. Pickering, and M. Senthilvelan, "The PrelleSinger method and Painlevé hierarchies," Journal of Mathematical Physics, vol. 55, no. 5, p. 053510, 2014. 